\title{
Dichromatic polynomials and Potts models summed over rooted maps
}

\author{
R.J. Baxter \\ School of Mathematical Sciences and Research School \\ of Physical Sciences \& Engineering \\ The Australian National University, Canberra, A.C.T. 0200, Australia
}

24 November 2000

\begin{abstract}
We consider the sum of dichromatic polynomials over non-separable rooted planar maps, an interesting special case of which is the enumeration of such maps. We present some known results and derive new ones. The general problem is equivalent to the $q$-state Potts model randomized over such maps. Like the regular ferromagnetic lattice models, it has a first-order transition when $q$ is greater than a critical value $q_{c}$, but $q_{c}$ is much larger - about 72 instead of 4 .
\end{abstract}

\section{Introduction}

The dichromatic polynomial of a graph $\mathcal{G}$ is a polynomial $\chi(\mathcal{G} ; x, y)$ in two variables $x, y$. It has been studied by Whitney 33], Tutte 27 and others. In particular, Tutte obtained a functional relation for the generating function $\Phi$ of the sum of dichromatic polynomials over rooted planar maps. 30

Fortuin and Kasteleyn 14, 18 showed, from the viewpoint of statistical physics, that $\chi(\mathcal{G} ; x, y)$ is equivalent to the partition function of a $q$-state Potts model on $\mathcal{G}$, with $q=(x-1)(y-1)$. This means that $\Phi$ is the partition function of the sum of maps. From it one should be able to obtain the thermodynamic properties of the planar Potts model, averaged over all rooted maps. This is not without interest: 
usually one considers such models on a regular lattice, but physical lattices are seldom (if ever) completely regular. They contain defects, and one should in fact average over such defects, i.e. over a lattice with some degree of randomness. In this sense $\Phi$ gives the average over over a completely random "lattice".

There has also been much work in statistical mechanics and field theory on summing over planar Feynman diagrams, using field-theoretic techniques and matrix models. 225, 9, 12, 19, 20] This appears to be a quite different form of randomization from the combinatorial approach that we discuss here.

Here we do try to reduce the randomness by restricting each map $\mathcal{G}$ to be non-separable (i.e. irreducible). The required analogue of Tutte's functional relation for this case has been obtained by Liu (eqn. 4.17 of [23]). There are three cases for which the relation can be solved explicitly:

Case 1: $x, y$ both large, of the same order;

Case 2: $x, y$ both small, of the same order;

Case 3: $q=(x-1)(y-1)=1$, or $x$ large and $y$ of order one, or $y$ large and $x$ of order one.

We present the solution in all three cases. The first is rather trivial. The second appears to be both non-trivial and new: one interesting feature we observe is that for non-separable maps $M$ of two or more edges

$$
\chi(M ; x, y) \sim c_{M}(x+y)+\text { higher terms in } x, y,
$$

the coefficients $c_{M}$ of $x$ and $y$ being the same positive integer. We first verified this on the computer for maps of up to ten edges. Since then Professor Tutte has pointed out to the author that (11) can be proved in general by recursively using Theorems 1 and 2 of Appendix A.

The last case is equivalent (to within a re-definition of $v_{1}, v_{2}$ ) to $\chi(\mathcal{G})=1$ for all maps $\mathcal{G}$, i.e. to a Potts model with no interaction (the ideal lattice gas). In this case the problem reduces to the enumeration of non-separable rooted planar maps. As the author discovered after solving this case, there is an extensive literature on this subject.26, 28, 32, 34, 21, 7, 11, 22, 2] Explicit results have been obtained by Tutte (eq. 6.4 of Ref. [26]) and Brown and Tutte. 10, 11] They involve the intriguing hypergeometric function identities (39), (40).

We also extend case 3 slightly, conjecturing the second-order corrections for $x$ large when $y=1$. 
In statistical mechanics we are particularly interested in large systems, when we expect the bulk properties to be obtainable by taking an appropriate limit. Combinatorially this corresponds to the asymptotic behaviour [8] of the sum of dichromatic polynomials. In all our solved cases we find that the appropriate limit does indeed exist.

For the regular planar lattices we know that the $q$-state Potts model has a first-order phase transition (i.e. a non-analyticity in the bulk properties) for $q>q_{c}$, and a continuous transition for $1<q \leq q_{c}$, where $q_{c}=4$. [4, 5, 6, 35] It seems from numerical sudies that the random model of this paper behaves similarly, but with $q_{c} \simeq 72$.

The outline of this paper is as follows. In the next section we define the dichromatic polynomial and the Potts model, and state their equivalence. Then we introduce the concept of non-separable rooted planar maps, and of the sum $\Phi$ over their dichromatic polynomials. We give the functional relation satisfied by $\Phi$. We then discuss the three explicitly solved cases, and go on to consider the asymptotic behaviour in the large-map limit. Finally we discuss the phase transition and numerical methods that can be used to study this.

\section{The dichromatic polynomial and the Potts model}

Let $\mathcal{G}$ be any connected graph (planar or not). Then the dichromatic polynomial is defined in [30] as

$$
\chi(\mathcal{G} ; x, y)=\sum_{S}(x-1)^{C(S)-1}(y-1)^{C(S)+E(S)-V(S)},
$$

where $x, y$ are arbitrary variables, the sum is over all sub-graphs $S$ of $\mathcal{G}$, and $C(S), E(S), V(S)$ are the numbers of connected components, edges and vertices, respectively, of $S$. Since $S$ and $\mathcal{G}$ share the same vertices, $V(S)=V(\mathcal{G})$. We have used Euler's relation: the number of circuits of $S$ is

$$
P(S)=C(S)+E(S)-V(S) .
$$

Thus for example the dichromatic polynomial of the graph of two vertices connected by a single edge is $(x-1)+(1)=x$; for a triangle of three vertices connected in pairs by three edges it is $(x-1)^{2}+3(x-$ $1)+3(1)+(y-1)=x^{2}+x+y$. 
A $q$-state Potts model is defined on $\mathcal{G}$ by associating with each vertex $i$ a "spin" $\sigma_{i}$ that takes the values $1, \ldots, q$. Let $K, v$ be two variables related by

$$
v=e^{K}-1
$$

Then the partition function is

$$
Z(\mathcal{G} ; q, v)=\sum_{\sigma} \exp \left\{K \sum_{(i, j)} \delta\left(\sigma_{i}, \sigma_{j}\right)\right\}
$$

where the outer sum is over all $q^{V(\mathcal{G})}$ values of all the spins, the inner sum is over all edges $(i, j)$ of $\mathcal{G}$, and $\delta(a, b)=1$ if $a=b, \delta(a, b)=0$ if $a \neq b$.

The eqn. (5) can be written as

$$
Z(\mathcal{G} ; q, v)=\sum_{\sigma} \prod_{(i, j)}\left\{1+v \delta\left(\sigma_{i}, \sigma_{j}\right)\right\}
$$

There are $E(\mathcal{G})$ factors in the product, one for every edge of $\mathcal{G}$. Expanding the product, we obtain $2^{E(\mathcal{G})}$ terms, each of which can be represented by a sub-graph $S$ of $\mathcal{G}$ by including an edge in $S$ if we take the $\left.v \delta\left(\sigma_{i}, \sigma_{j}\right)\right\}$ term, leaving it out if we take the leading term 1 .

For each of the $2^{E(\mathcal{G})}$ terms thus obtained, we can readily perform the sum over the spins, giving

$$
Z(\mathcal{G} ; q, v)=\sum_{S} q^{C(S)} v^{E(S)}
$$

so we see that

$$
Z(\mathcal{G} ; q, v)=(x-1)(y-1)^{V(\mathcal{G})} \chi(\mathcal{G} ; x, y)
$$

provided that

$$
q=(x-1)(y-1), \quad v=y-1 .
$$

\section{Non-separable rooted planar maps}

A planar map (or simply a map) is a connected graph embedded in the surface of a sphere or closed plane, with no crossing edges. Not all graphs are planar, and those that are may correspond to more than one planar map: for instance the maps in Figure 11 are different, but correspond to the same graph. If the vertices and faces are given 
distinctive labels, then a map can be specified uniquely by listing the anti-clockwise cyclic sequence of alternating vertices and faces (two of each) round each edge. Two maps are distinct if and only if their edge lists cannot be made the same by re-labelling the vertices and sites, and re-ordering the list.
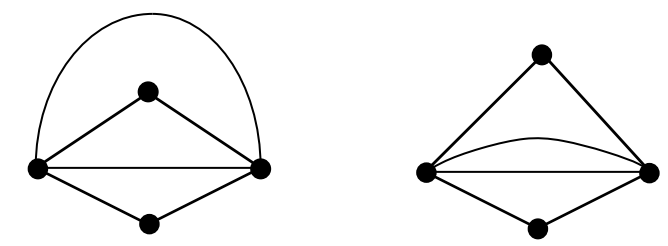

Figure 1: Two maps corresponding to the same graph.

A map $M$ is rooted if one edge $A$ is chosen as the root-edge and given a direction. The vertex at the start of this directed edge is known as the root-vertex, the face on the left as the root-face. Two rooted maps are distinct if and only if their edge lists, root-edge, root-vertex and root-face cannot all be made the same by re-labelling the vertices and sites, and re-ordering the list.

Finally, a graph is non-separable if there is no vertex such that the graph obtained by cutting it at this vertex (i.e. disconnecting from the vertex all its incident edges) is no longer connected. The nonseparable rooted maps of up to three edges are shown in Figure 2. Their dichromatic polynomials, as defined by (2), are $x, y, x+y, x^{2}+$ $x+y, x+y+y^{2}$, respectively. The first two are the link-map and the loop-map: they are the smallest maps we shall consider. Note that we allow repeated edges, but only the loop-map contains a loop, and only the link-map has sites of valence one.

Here we follow the original notation of Tutte[30] rather than Liu. 23] For a given map $M$, let $m, n, i, j$ be the valency of the root-face, the valency of the root-vertex, the number of faces other than the rootface, and the number of vertices other than the root-vertex. Then the number of edges of $M$ is $i+j$. We say that a map is "of type $(m, n, i, j) "$ if it has these values of $m, n, i, j$. Define

$$
\Phi=\Phi\left(u_{1}, u_{2}\right)=\Phi\left(u_{1}, u_{2}, v_{1}, v_{2}, x, y\right)
$$




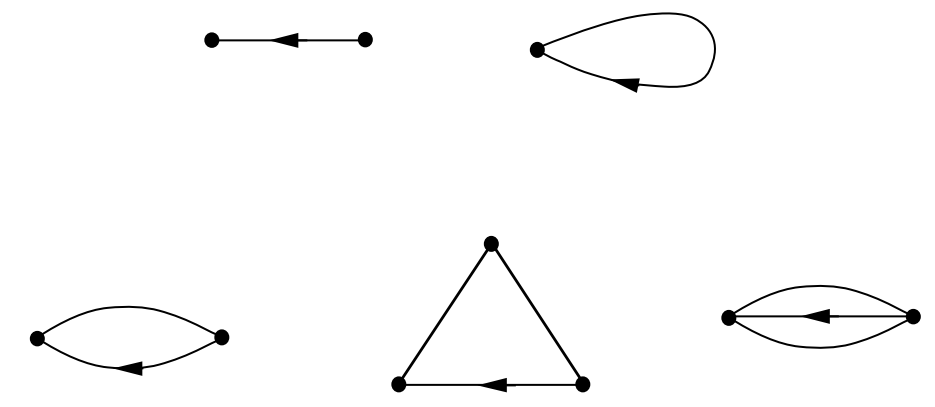

Figure 2: Non-separable rooted maps with up to three edges.

$$
=\sum_{M} u_{1}^{m} u_{2}^{n} v_{1}^{i} v_{2}^{j} \chi(M ; x, y),
$$

where the sum is over all distinct rooted non-separable maps $M$ and $m, n, i, j$ are the numbers defined above for each map $M$. Writing the contributions from the link and loop maps explicitly, this is

$$
\Phi\left(u_{1}, u_{2}\right)=x u_{1}^{2} u_{2} v_{2}+y u_{1} u_{2}^{2} v_{1}+\Phi^{\prime}\left(u_{1}, u_{2}\right),
$$

where the sum $\Phi^{\prime}\left(u_{1}, u_{2}\right)$ is further restricted to maps of two or more edges. Such maps also have $i, j \geq 1$ and $m, n \geq 2$, so

$$
\Phi^{\prime}\left(u_{1}, u_{2}\right)=\sum_{m, n, i, j} N(m, n, i, j) u_{1}^{m} u_{2}^{n} v_{1}^{i} v_{2}^{j}
$$

where

$$
1 \leq m-1 \leq j, \quad 1 \leq n-1 \leq i \quad .
$$

The coefficient $N(m, n, i, j)$ is the sum of the dichromatic polynomials over all distinct non-separable rooted maps of type $(m, n, i, j)$. We also define coefficients $N^{\prime}, P, R$ by

$$
\begin{aligned}
\Phi^{\prime}\left(1, u_{2}\right) & =\sum_{n, i, j} N^{\prime}(n, i, j) u_{2}^{n} v_{1}^{i} v_{2}^{j}, \\
\Phi^{\prime}(1,1) & =\sum_{i, j} P(i, j) v_{1}^{i} v_{2}^{j} . \\
\Phi^{\prime}(1,1, v, v, x, y) & =\sum_{k=2}^{\infty} R(k) v^{k} .
\end{aligned}
$$

Thus $P(i, j)$ is the sum over all distinct non-separable rooted maps with $i+1$ faces and $j+1$ vertices; $R(k)$ is the sum over all such maps 
with $k$ edges. Clearly

$$
\begin{gathered}
N^{\prime}(n, i, j)=\sum_{m=2}^{j+1} N(m, n, i, j) \\
P(i, j)=\sum_{n=2}^{i+1} N^{\prime}(n, i, j), \quad R(k)=\sum_{i=1}^{k-1} P(i, k-i)
\end{gathered}
$$

From Figure 2 and the comments above,

$\Phi^{\prime}\left(u_{1}, u_{2}\right)=(x+y) u_{1}^{2} u_{2}^{2} v_{1} v_{2}+\left(x^{2}+x+y\right) u_{1}^{3} u_{2}^{2} v_{1} v_{2}^{2}+\left(x+y+y^{2}\right) u_{1}^{2} u_{2}^{3} v_{1}^{2} v_{2}+\cdots$

all other terms being of order $v_{1}^{i} v_{2}^{j}$, with $i+j>3$.

Tutte shows that the concept of duality extends to rooted maps. If $M^{*}$ is the dual of $M$, and $m^{*}, n^{*}, i^{*}, j^{*}$ the corresponding values of $m, n, i, j$, then

$$
m^{*}=n, n^{*}=m, i^{*}=j, j^{*}=i,
$$

and

$$
\chi(M ; x, y)=\chi\left(M^{*} ; y, x\right) .
$$

The dual of a non-separable map is also non-separable, so

$$
\Phi\left(u_{1}, u_{2}, v_{1}, v_{2}, x, y\right)=\Phi\left(u_{2}, u_{1}, v_{2}, v_{1}, y, x\right)
$$

We can use this duality to obtain the sum over $n$ of $N(m, n, i, j)$ from $N^{\prime}$.

Define

$$
\begin{aligned}
& C\left(u_{1}, u_{2}\right)=\frac{u_{1} \Phi\left(1, u_{2}\right)-\Phi\left(u_{1}, u_{2}\right)}{1-u_{1}}, \\
& D\left(u_{1}, u_{2}\right)=\frac{u_{2} \Phi\left(u_{1}, 1\right)-\Phi\left(u_{1}, u_{2}\right)}{1-u_{2}} .
\end{aligned}
$$

Then Liu[23] shows that $\Phi$ satisfies the functional relation

$$
\begin{gathered}
\Phi\left(u_{1}, u_{2}\right)=x u_{1}^{2} u_{2} v_{2}+y u_{1} u_{2}^{2} v_{1}+\frac{u_{1} u_{2} v_{1} C\left(u_{1}, u_{2}\right)}{1-C\left(u_{1}, 1\right)} \\
+\frac{u_{1} u_{2} v_{2} D\left(u_{1}, u_{2}\right)}{1-D\left(1, u_{2}\right)} .
\end{gathered}
$$

For completeness we give the derivation of this result in Appendix A. 
We can solve this equation iteratively to obtain the contributions to $\Phi$ from non-separable rooted maps of $1,2,3, \ldots$ edges. As we shall find in the next section, the numbers of such distinct maps are 2,1,2,6, 22, 91, 408, 1938, 9614, 49335,... (e.g. there are 91 non-separable rooted maps with six edges).

As a check, we have enumerated all non-separable maps of up to 10 edges, and their various rootings and dichromatic polynomials, directly on the computer. We have verified that the results for $\Phi$ do in fact agree with those obtained from (20). We also find that the numbers of distinct unrooted maps are 2,1,2,3,6, 16, 42, 151, 596, $2605, \ldots$ The problem of counting unrooted maps has quite an extensive literature. Wormald [34, and Liskovets and Walsh [21], established linear relations between the numbers of unrooted and rooted maps: both the above sequences of integers appear in Figure 1 of Ref. 21. The relation between them is given in Appendix B.

\section{Case 1: $x, y$ both large}

One case that can be easily handled is when $x, y$ are both large and of the same order. In this case the coefficients in $\Phi$ of $v_{1}^{i} v_{2}^{j}$, for $i, j \geq 1$ and arbitrary $u_{1}, u_{2}$, are of order $i+j+\delta_{i 1}+\delta_{j 1}-2$. To obtain them it is sufficient to set $v_{1}=w_{1} / y, v_{2}=w_{2} / x$, take $w_{1}, w_{2}$ to be fixed and of order one, and then to solve (20) iteratively in inverse powers of $x$ and $y$. To first order the the result is

$$
\Phi\left(u_{1}, u_{2}\right)=u_{1}^{2} u_{2} w_{2}+u_{1} u_{2}^{2} w_{1}+y^{-1} \frac{u_{1}^{2} u_{2}^{2} w_{1} w_{2}}{1-u_{1} w_{2}}+x^{-1} \frac{u_{1}^{2} u_{2}^{2} w_{1} w_{2}}{1-u_{2} w_{1}}+\cdots
$$

Continuing to second order, then setting $u_{1}=u_{2}=1$, we obtain

$$
\begin{gathered}
\Phi(1,1)=w_{1}+w_{2}+\mu_{1} w_{1} w_{2} / x+w_{1} \mu_{2} w_{2} / y+\mu_{1}^{4} w_{1} w_{2}^{2} / x^{2}+ \\
\mu_{1}^{2} w_{1} \mu_{2} w_{2}^{2} /(x y)+\mu_{1} w_{1}^{2} \mu_{2}^{2} w_{2} /(x y)+w_{1}^{2} \mu_{2}^{4} w_{2} / y^{2}+\cdots
\end{gathered}
$$

where for brevity we have written $1 /\left(1-w_{i}\right)$ as $\mu_{i}$. From this and (14) it follows that if $i=1$ or $j=1$

$$
P(i, j) \sim x^{j} \delta_{i 1}+y^{i} \delta_{j 1}
$$

if $i, j \geq 2$

$$
P(i, j) \sim(i+j) x^{j-1} y^{i-1}+\left(\begin{array}{c}
i+j \\
3
\end{array}\right)\left(x^{j} \delta_{i 2}+y^{i} \delta_{j 2}\right)
$$


and

$$
R(k) \sim x^{k-1}+y^{k-1} .
$$

\section{Case 2: $x, y$ small}

For any graph, the dichromatic polynomial vanishes when $x=y=$ 0 . Consider the limit when $x, y$ are small but non-zero, of the same order. Then any dichromatic polynomial is at most of this order. From (10) and (19), so are $\Phi, C$ and $D$. If we define $Q=Q\left(u_{1}, u_{2}\right)=$ $Q\left(u_{1}, u_{2}, v_{1}, v_{2}\right)$ by

$$
\Phi\left(u_{1}, u_{2}\right)=x u_{1}^{2} u_{2} v_{2}+y u_{1} u_{2}^{2} v_{1}+(x+y) u_{1}^{2} u_{2}^{2} v_{1} v_{2} Q\left(u_{1}, u_{2}\right),
$$

then we find from (20) that $x, y$ cancel out of the functional relation for $Q$, leaving

$$
\begin{aligned}
Q\left(u_{1}, u_{2}\right)= & 1+u_{2} v_{1} \frac{u_{1} Q\left(u_{1}, u_{2}\right)-Q\left(1, u_{2}\right)}{u_{1}-1}+ \\
& u_{1} v_{2} \frac{u_{2} Q\left(u_{1}, u_{2}\right)-Q\left(u_{1}, 1\right)}{u_{2}-1} .
\end{aligned}
$$

This implies that, apart from the contributions of the link-map and the loop-map, $\Phi\left(u_{1}, u_{2}, v_{1}, v_{2}\right)$ is proportional to $x+y$ when $x$ and $y$ are both small. As we state in the introduction, the stronger statement (1) is true, for every non-separable map $M$ of more than one edge. For all maps of two to five edges $c_{M}=1$, for six or seven edges it is 1 or 2 , for eight to ten edges it is no bigger than the number of edges minus five (i.e. $3,4,5$, respectively). The first map to have $c_{M}>1$ is the tetrahedron (which is planar).

The relation (25) is a linear equation for $Q\left(u_{1}, u_{2}\right)$. If we regard $Q\left(1, u_{2}\right)$ and $Q\left(u_{1}, 1\right)$ as known, we can solve it for $Q\left(u_{1}, u_{2}\right)$. The result is a rational expression with a denominator that is biquadratic in $u_{1}, u_{2}$. If $v_{1}, v_{2}$ are both small and $u_{2}$ is of order one, the two zeros are when $u_{1} \simeq 1$ and when $u_{1} \simeq 1 /\left(u_{2} v_{2}\right)$.

Suppose $v_{1}, v_{2}$ are both small, of order $v$. From (13), $m \leq i+j$ (for $i+j \geq 2$ ), so the coefficient of $u_{1}^{m}$ in the expansion of $\Phi$ is of order $v^{m}$ or smaller. It follows that the zero near $u_{1}=1$ must also be a zero of the numerator, since otherwise it would be a pole of $\Phi$ and the coefficient of $u_{1}^{m}$ would be of order unity.

This gives a linear relation between $Q\left(1, u_{2}\right)$ and $Q\left(u_{1}, 1\right)$ at this value of $u_{1}$. We have not yet found a direct way to solve this relation, 
but we have guessed explicit expressions for the coefficients in the expansion of $Q\left(u_{1}, u_{2}\right)$. In terms of the coefficients $N, N^{\prime}, P$ of (12), (14), they are:

$$
\begin{gathered}
N(m, n, i, j)=(x+y)(m-1)(n-1) \lambda(m, n, i, j) \times \\
\frac{(i+j-2) !(i+j-m-1) !(i+j-n-1) !}{(i-1) ! i !(i-n+1) !(j-1) ! j !(j-m+1) !} \\
N^{\prime}(n, i, j)=\frac{(x+y)(n-1) n(i+j-2) !(i+j-1) !(i+j-n-1) !}{i !(i+1) !(i-n+1) !(j-2) !(j-1) ! j !} \\
P(i, j)=\frac{2(x+y)(i+j-2) !(i+j-1) !(i+j) !}{(i-1) ! i !(i+1) !(j-1) ! j !(j+1) !}
\end{gathered}
$$

where

$$
\lambda(m, n, i, j)=i m+j n-m n+m+n-2 i-2 j .
$$

These formulae hold for all $m, n, i, j$ satisfying (13) and $i, j \geq 2$. The last, for $P(i, j)$, extends to $i, j \geq 1$, while the first two should be supplemented with

$$
\begin{gathered}
N(m, n, i, 1)=(x+y) \delta_{m 2} \delta_{n, i+1}, \quad N(m, n, 1, j)=(x+y) \delta_{n 2} \delta_{m, j+1} \\
N^{\prime}(n, 1, j)=(x+y) \delta_{n 2}, \quad N^{\prime}(n, i, 1)=(x+y) \delta_{n, i+1}
\end{gathered}
$$

for $i, j \geq 1$.

It is straightforward to prove that these guesses are correct by direct substitution into (24) and (25). One needs the symmetry $N(m, n, i, j)=$ $N(n, m, j, i)$ and the identity

$$
\sum_{k=m}^{j+1} N(k, n, i, j)=\frac{(i+j-m) B(m, n, i, j) N(m, n, i, j)}{i(i+1)(m-1) \lambda(m, n, i, j)},
$$

where

$$
B(m, n, i, j)=(m-2)(m-1) i(i-n+1)+(j-1) n(i m-m-i+j+1) \quad .
$$

The identity (27) can be verified by taking the difference between its $m$ and $m-1$ cases. 


\section{Case 3: enumeration of rooted non-separable maps}

If

$$
q=(x-1)(y-1)=1 .
$$

Then the dependence on $C(S)$ in (2) disappears and we immediately obtain (for all graphs)

$$
\chi(\mathcal{G} ; x, y)=y^{E}(\mathcal{G}) /(y-1)^{V(\mathcal{G})+1} .
$$

For our maps $M, E(M)=i+j$ and $V(M)=j+1$, so

$$
\chi(M ; x, y)=x^{j} y^{i} .
$$

Hence $x$ and $y$ can be absorbed into $v_{2}$ and $v_{1}$ and

$$
\Phi^{\prime}\left(u_{1}, u_{2}, v_{1}, v_{2}, x, y\right)=T\left(u_{1}, u_{2}, y v_{1}, x v_{2}\right),
$$

where

$$
T\left(u_{1}, u_{2}, v_{1}, v_{2}\right)=T\left(u_{1}, u_{2}\right)=\sum_{M} u_{1}^{m} u_{2}^{n} v_{1}^{i} v_{2}^{j}
$$

Thus the coefficient in $T\left(u_{1}, u_{2}\right)$ of $u_{1}^{m} u_{2}^{n} v_{1}^{i} v_{2}^{j}$ is simply the number of rooted non-separable maps of type $(m, n, i, j)$.

This case was solved originally by Brown and Tutte [10, 11], and has been further investigated by a number of authors, including Arques [1, 2], Bender [7, 8], Liskovets and Walsh [32, 21], Liu [22, 23] and Wormald [34].

As a combinatoric problem this case is of interest in itself. From the point of view of statistical mechanics it is very simple, corresponding to the one-state Potts model or (as we show below) a q-state model with no interactions, i.e. the perfect gas. Even so, we hope to use it as the first step in examining the interacting Potts model of our random model, and we certainly do need to understand it if we are to use it as a starting point for general values of $x$ and $y$. We therefore present the results here and outline the working in Appendix C. It involves some rather surprising identities.

$\mathrm{C}$ and D simplify in the same way as $\Phi^{\prime}: C\left(u_{1}, u_{2}, v_{1}, v_{2}, x, y\right)=$ $\hat{C}\left(u_{1}, u_{2}, y v_{1}, x v_{2}\right)$ and $D\left(u_{1}, u_{2}, v_{1}, v_{2}, x, y\right)=\hat{D}\left(u_{1}, u_{2}, y v_{1}, x v_{2}\right)$. The definitions (19) become

$$
\hat{C}\left(u_{1}, u_{2}, v_{1}, v_{2}\right)=u_{1} u_{2} v_{2}+\frac{u_{1} T\left(1, u_{2}, v_{1}, v_{2}\right)-T\left(u_{1}, u_{2}, v_{1}, v_{2}\right)}{1-u_{1}},
$$




$$
\hat{D}\left(u_{1}, u_{2}, v_{1}, v_{2}\right)=u_{1} u_{2} v_{1}+\frac{u_{2} T\left(u_{1}, 1, v_{1}, v_{2}\right)-T\left(u_{1}, u_{2}, v_{1}, v_{2}\right)}{1-u_{2}}
$$

Replacing $v_{1}, v_{2}$ in (20) by $v_{1} / y, v_{2} / x$ and using these functions $T, \hat{C}, \hat{D}$, we find that $x, y$ cancel out, except only that the last two terms (involving $C$ and $D$, respectively) acquire factors $1 / y, 1 / x$. This is the only place $x$ and $y$ now appear. Since the relation must hold for all $x, y$ satisfying (28), and this can be written as $1 / x+1 / y=1$, we obtain two relations from (20), namely

$$
T\left(u_{1}, u_{2}\right)=\frac{u_{1} u_{2} v_{1} \hat{C}\left(u_{1}, u_{2}\right)}{1-\hat{C}\left(u_{1}, 1\right)}=\frac{u_{1} u_{2} v_{2} \hat{D}\left(u_{1}, u_{2}\right)}{1-\hat{D}\left(1, u_{2}\right)} .
$$

Other ways to reduce the dichromatic problem to simple enumeration are to allow $x$ or $y$ to tend to infinity, keeping the other fixed. For instance, when $x$ is large the sum in (2) is dominated by sub-graphs $S$ where $C(S)$ attains its maximum value $V(S)$. For all but the loopmap this means the sub-graph with no edges, so $\chi(M ; x, y) \sim x^{j}$ and we can absorb $x$ into $v_{2}$. We obtain (34), but without the last equality involving $\hat{D}$. The resulting relation is still sufficient to iteratively determine $T\left(u_{1}, u_{2}\right)$, and hence its desired power series coefficients, but it is not as full a description of the properties.

These equations are fully solved in parametric form in Appendix C. In particular, we introduce two new variables $p, r$, defined in terms of $v_{1}, v_{2}$ by

$$
v_{1}=p(1-r)^{2}, \quad v_{2}=r(1-p)^{2} .
$$

Then

$$
T(1,1)=p r(1-p-r) .
$$

These equations are given in section 2 of [11], our $v_{1}, v_{2}, p, r$ being the $x, y, u, v$ therein. As is noted in section 4 therein, products of powers of $p$ and $r$ (and hence of $1-p$ and $1-r$ ) have remarkably simple expansions in powers of $v_{1}$ and $v_{2}$. Let us introduce the standard notation

$$
\begin{aligned}
(m)_{k} & =m(m+1)(m+2) \cdots(m+k-1) \text { for } k>0, \\
& =1 \text { for } k=0, \\
& =1 /[(m+k)(m+k+1) \cdots(m-1)] \text { for } k<0,
\end{aligned}
$$


where $k$ is an integer.

For positive integers $i, j$, define

$$
\beta(m, n \mid i, j)=\frac{4(n i+m j+m n)(2 i+2 m+1)_{j-1}(2 j+2 n+1)_{i-1}}{i ! j !} .
$$

This can be extended to $i$ or $j$ zero by taking an appropriate limit, giving $\beta(m, n \mid 0, j)=2 m(2 m+1)_{j-1} / j !, \beta(m, n \mid i, 0)=2 n(2 n+1)_{i-1} / i$, and $\beta(m, n \mid 0,0)=1$. Thus $\beta(m, n \mid i, j)$ is a polynomial in $m$ and $n$, for all non-negative integers $i, j$.

Then we find the following expansion in powers of $v_{1}, v_{2}$ :

$$
p^{m} r^{n}=\sum_{i=0}^{\infty} \sum_{j=0}^{\infty} \beta(m, n \mid i, j) v_{1}^{i+m} v_{2}^{j+n} .
$$

This elegant formula is true for all integers $m, n$, positive or negative, and can be extended to real and complex values of $m, n$.

If $v_{1}=v_{2}=v$, then $r=p$ and

$$
p^{m}=\sum_{k=0}^{\infty} \alpha(m, k) v^{k+m},
$$

where

$$
\alpha(m, k)=2 m(2 k+2 m+1)^{k-1} / k ! .
$$

This is also an intriguing result.

The coefficients $P(i, j), R(k)$ in (14) are now easily determined:

$$
P(i, j)=4 y^{i} x^{j} \frac{4(i+2 j-2) !(2 i+j-2) !}{(i-1) !(2 i) !(j-1) !(2 j) !},
$$

and if $x=y$,

$$
R(k)=x^{k} \frac{2(3 k-3) !}{k !(2 k-1) !} .
$$

Thus $P(i, j) /\left(y^{i} x^{j}\right)$ is the number of non-separable rooted maps with $i+1$ faces and $j+1$ vertices, while $R(k) / x^{k}$ is the number with $k$ edges. These formulae were first given by Tutte and Brown [26, 11].

We have not found an explicit formula for the coefficients $N^{\prime}(n, i, j)$, $N(m, n, i, j)$, but it does follow from Appendix $\mathrm{C}$ that they have a certain structure. Let $m^{\prime}=m-1+\delta_{m 2}, n^{\prime}=n-1+\delta_{n 2}, m^{\prime \prime}=m-\delta_{m 2}-2$ and $n^{\prime \prime}=n-\delta_{n 2}-2$. Then

$$
N(m, n, i, j)=\frac{4 y^{i} x^{j}(2 i-1)_{j-2 m-n^{\prime \prime}+2}(2 j-1)_{i-m^{\prime \prime}-2 n+2} G_{m n}(i, j)}{\left(i-n^{\prime}\right) !\left(j-m^{\prime}\right) !}
$$




$$
N^{\prime}(n, i, j)=\frac{4 y^{i} x^{j}(2 i+1)_{j-n-1}(2 j-1)_{i-2 n+2} G_{n}^{\prime}(i, j)}{(i-n+1) !(j-2) !} .
$$

Here $G_{m n}(i, j)$ and $G_{n}^{\prime}(i, j)$ are polynomials in $i, j$ of total degree $2 m^{\prime \prime}+2 n^{\prime \prime}, 2 n-4$, respectively. By "of total degree $d$ ", we mean that the sum of the powers of $i$ and $j$ in any term does not exceed $d$. In particular, $G_{22}(i, j)=0, G_{23}(i, j)=1, G_{2,4}(i, j)=3(21-15 i+$ $\left.2 i^{2}-17 j+5 i j+4 j^{2}\right), G_{3,3}(i, j)$ is of total degree $4, G_{2}^{\prime}(i, j)=1$ and $G_{3}^{\prime}(i, j)=6 i^{2}+3 i(5 j-11)+12(j-1)(j-2)$.

The coefficients $N(m, n, i, j), N^{\prime}(n, i, j)$ are always finite. For small values of $i, j$ the factors in (44) other than $G_{m n}(i, j), G_{n}^{\prime}(i, j)$ may become infinite, but in this case $G_{m n}(i, j)$ or $G_{n}^{\prime}(i, j)$ vanishes and the coefficient can be evaluated by taking an appropriate limit. This is quite a strong constraint on the polynomials, but not apparently strong enough to determine them.

\section{The large-graph limit}

In statistical mechanics we are usually interested in the "thermodynamic limit", when the system becomes large in such a way that bulk properties, such as the total free energy or total entropy become proportional to the size of the system, and local or average properties, such as the density, tend to a limit.

For any regular lattice the ratio of the number of faces to the number of vertices tends to a limit, determined by the valency. For the honeycomb, square and triangular lattices this ratio is $\frac{1}{2}, 1,2$, respectively. For any self-dual map it is one. This suggest that we should consider the limit when $i, j$ become large, their ratio remaining fixed, non-zero and finite. This can be done in a symmetrical way by defining

$$
k=i+j, i=k \alpha, j=k \beta,
$$

and holding $\alpha, \beta$ fixed while $k \rightarrow \infty$. Note that $\alpha+\beta=1$.

In all the cases discussed above we find that the coefficients $N(m, n, i, j), N^{\prime}(n, i, j), P(i, j), R(k)$ are asymptotically of the form

$$
\text { constant } \times \mathcal{K}^{k} / k^{c}
$$

i.e. their ratio to $\mathcal{K}^{k} / k^{c}$ tends to a non-zero limit as $k \rightarrow \infty$. This $\mathcal{K}$ is the "partition function per site" and is in general a function of $\alpha, \beta, x, y ; c$ is an exponent, which for all our cases is a simple rational number. 


\begin{tabular}{|cccc|}
\hline Case & Coeffs & $\mathcal{K}$ & $c$ \\
\hline 1 & $P$ & $x^{\beta} y^{\alpha}$ & -1 \\
1 & $R$ & $\max (x, y)$ & 0 \\
2 & $N, N^{\prime}, P$ & $\alpha^{-3 \alpha} \beta^{-3 \beta}$ & $9 / 2$ \\
2 & $R$ & 8 & 4 \\
3 & $N, N^{\prime}, P$ & $\lambda^{\alpha} \mu^{\beta}$ & 3 \\
3 & $R$ & $27 / 4$ & $5 / 2$ \\
\hline
\end{tabular}

Table 1: Values of $\mathcal{K}$ and $c$ for the coefficients $N, N^{\prime}, P, R$ of the three solved cases.

Define

$$
\begin{aligned}
& \lambda=(1+\alpha)^{2}(1+\beta) /\left(4 \alpha^{3}\right), \\
& \mu=(1+\alpha)(1+\beta)^{2} /\left(4 \beta^{3}\right) .
\end{aligned}
$$

Then the results for $\mathcal{K}$ and $c$ are summarised in Table 1.

For both cases 2 and $3, \mathcal{K}$ and $c$ are the same for the coefficients $N, N^{\prime}, P$, provided we keep $m, n$ fixed (or at least small compared with $k$ ) while we take the limit $k \rightarrow \infty$. Thus $m, n$ affect only the proportionality factor in (46): from the viewpoint of statistical mechanics they are "boundary effects" corresponding to various weightings of the rootings.

It is not actually necessary to calculate the coefficients to obtain $\mathcal{K}$. From (14) and (46), the radius of convergence of the series for $\Phi^{\prime}(1,1, v, v, x, y)$ in powers of $v$ is $1 / \mathcal{K}$, so $1 / \mathcal{K}$ is the position of the closest singularity to the origin in the complex $v$-plane of $\Phi^{\prime}(1,1, v, v, x, y)$. For instance, for case 3 this is where the relation (35), with $v_{1}=v_{2}, r=p$, i.e. $v=p(1-p)^{2}$ ceases to be invertible. This is when $d v / d p=0$, i.e. $p=1 / 3, v=4 / 27$, so $\mathcal{K}=27 / 4$, as in Table 1.

Similarly, if $v_{1} \neq v_{2}$ and $p \neq r$, then the singularity is when the Jacobian $\partial\left(v_{1}, v_{2}\right) / \partial(p, r)$ vanishes, which is when $3 p r+p+r=1$, i.e. when $r=(1-p) /(1+3 p)$. Thus

$$
v_{1}^{i} v_{2}^{j}=\left(\frac{16 p^{3}}{(1+3 p)^{2}}\right)^{k \alpha}\left(\frac{(1-p)^{3}}{1+3 p}\right)^{k \beta} .
$$


For given $\alpha, \beta$, this is maximized when $p$ is chosen so that

$$
p=\alpha /(\alpha+2 \beta), r=\beta /(2 \alpha+\beta),
$$

so then

$$
v_{1}^{i} v_{2}^{j}=\lambda^{-k \alpha} \mu^{-k \beta},
$$

where $\lambda, \mu$ are defined as above. From Table 1 , this is $\mathcal{K}^{-k}$, so the double series (14) for $\Phi^{\prime}(1,1)$ then just fails to converge, as of course it should.

Further discussion of the techniques available to handle the asymptotic behaviour in case 3 is given by Bender and Richmond. [8]

\section{A conjectured extension of case 3: $y=1$ and $x$ large}

Ultimately it seems we should try to expand about case 3 . We noted in that section that there are actually three sub-cases that reduce to the enumeration problem, namely $x+y=x y, x$ large and $y$ arbitrary, and $y$ large and $x$ arbitrary. We can consider an expansion about the second sub-case, expanding in inverse powers of $x$ while keeping $y$ fixed. If we choose $y=1$, then we have observed a pattern in the coefficients $P(i, j)$, namely that to second-next-to-leading order the RHS of (42) becomes

$$
4 x^{j} \frac{4(i+2 j-2) !(2 i+j-2) !}{(i-1) !(2 i) !(j-1) !(2 j) !}\left(1+\frac{i}{x}+\frac{i(i+1)+2 h}{2 x^{2}}+O\left(x^{-3}\right)\right),
$$

where

$$
h=\frac{i(i-1)(2 i-1)(i+j)\left(17-9 i+3 j-3 i j-2 j^{2}\right)}{(j+1)(2 j+1)(2 i+j-2)(2 i+j-3)(2 i+j-4)} .
$$

We conjecture that this is correct for all $i, j \geq 1,2 i+j>4$. It does have the expected behaviour (46) in the asymptotic limit. The partition function per site $\mathcal{K}$ is modified, acquiring an extra factor

$$
1+\frac{\alpha}{x}+\frac{\alpha+\alpha^{2}}{2 x^{2}}-\frac{\alpha^{3}(\alpha+2)}{\beta(\alpha+1)^{3} x^{2}}+\cdots
$$

(remember that $\alpha+\beta=1$ ). To this order the exponent $c$ remains at $c=3$ : there are no logarithmic terms in $k$ that would imply a variation in this exponent. 17] 


\section{Summary}

The functional relations developed by Tutte [30] and Liu [23] give equations that define the sum of dichromatic poynomials over rooted non-separable planar maps. This is the partition function of the Potts model, summed (i.e. randomized) over such maps.

We are interested in the partial sum over maps with a given number of sites and vertices $(i+1$ and $j+1$, respectively), or with a given number $k=i+j$ of edges. We are particularly interested in the "thermodynamic" limit when $i, j, k$ all become large, the ratios $\alpha=i / k, \beta=j / k$ remaining finite. We then expect the partial sums to behave asymptotically as (46). The quantity $\mathcal{K}$ therein is the "partition function per edge". We expect it to depend on $x, y, \alpha, \beta$ (or just $x, y$ if it is derived from $R(k)$ ), but not on the parameters $m, n$ which relate to the way in which the maps are rooted. For this reason we would be happy to restrict attention to the case $u_{1}=u_{2}=1$ (or any other fixed values of $u_{1}, u_{2}$ ), but we need to keep them arbitrary for the functional relations (20) as written to define the sum $\Phi$.

For the regular square lattice we know that the limiting function $\mathcal{K}(x, y)$ has a singularity at $x=y$. For $x>3$ (i.e. $q>4$ ) this takes the form of a discontinuity in $\partial \mathcal{K} / \partial x$ as one crosses the line $x=y$. For $1<x \leq 3(0<q \leq 4)$ there is no discontinuity, but there is a non-analyticity. Physically this means that the Potts model has a phase transition at the self-dual point $x=y$, which is first order for $q>4$, continuous for $1<q \leq 4$. 囷, 35]

The same is true for the triangular and honeycomb lattices, except that because these lattices are not self-dual, the transition is now at the point which maps to itself under a duality plus a star-triangle transformation. For the triangular (honeycomb) lattice this is when $y^{2}+y=x+1\left(x^{2}+x=y+1\right)$. [5]

The obvious question is whether the $\mathcal{K}$ of this paper has similar properties, or whether the transition is removed by the randomization introduced by summing over maps. For the regular lattices one can develop expansions for large $x$ and $y$ that make it quite clear that there is a first order transition. Unfortunately this technique fails for our randomization. From Table 1 the exponent $c$ is different for the three solved cases that we have presented. There are two explanations for this: either it varies continuously from case to case, or it varies discontinuously. We conjecture that the latter is true: all the values presented are integers or half-integers, and it seems likely that this is 
always true. Cases 1 and 2 are the large and small $x, y$ limits, and it is likely that one is not allowed to interchange these limits with the large- $k$ asymptotic limit. This suggests that $c$ has the values of case 3 (namely 3 and 5/2) for all positive finite $x$ and $y$, and this agrees with the second-order expansion of the previous section.

So to investigate the phase transition problem we should focus on finite positive $x, y$ and look to see if $\mathcal{K}(x, y, \alpha, \beta)$ has non-analyticities for positive $x, y, \alpha, \beta$, in particular whether it is singular across the self-dual line $x=y$ when $\alpha=\beta$.

In one sense the problem is solved: one can iteratively solve (20) in increasing powers of $v_{1}$ and $v_{2}$, the computing needed growing polynomially with the number of terms. A.J. Guttmann and others have argued that this is effectively a solution: it is exponential growth (which is usual in statistical mechanical problems) that can make a problem intractable numerically.

Of course the problem with this is that one needs to take the limit of high powers of $v_{1}$ and $v_{2}$ in order to evaluate $\mathcal{K}$. Any finite powers will only give a numerical approximation to $\mathcal{K}$, and it is notoriously difficult to determine non-analyticities from numerical approximations. One could focus on the case $v_{1}=v_{2}=v$, thereby calculating $R(k)$, and assign numerical values to $x$ and $y$. This would still leave one with the problem of handling a series expansion in the three variables $u_{1}, u_{2}, v$, so the calculation of $R(k)$ would grow with $k$ as $k^{3}$. It may be possible to make progress in this way, but it would certainly be a major numerical exercise.

One could reduce the problem to one of working with functions of a single variable $u_{1}$ or $u_{2}$ (but not both) by generalizing the arguments given after (25), which would reduce the memory problems in a numerical calculation. It would of course be even better to remove both $u_{1}$ and $u_{2}$, or even obtain explicit equations for the asymptotic behaviour in the limit $k \rightarrow \infty$. This can be done in case 3: can it be done in general?

Since writing these last paragraphs we have numerically investigated the sum of $P(i, k-i)$ in (15). If $x=y$, then $P(i, j)=P(j, i)$, so the terms in the sum are symmetrical about $i=k / 2$. For sufficiently small $x$ there is a single maximum, at $i=k / 2$, and this dominates the sum in the limit of $k$ large. For sufficiently large $x$ there are two maxima, symmetric about $i=k / 2$, which together dominate the sum. If $x$ is slightly different from $y$, one will be larger than the other. As $k$ increases, both will grow exponentially, and the larger will by itself 
dominate the sum. Hence one can define an order parameter:

$$
M(x)=\lim _{x \rightarrow y^{+}} \lim _{k \rightarrow \infty} \sum_{i=1}^{i=k-1}(k-2 i) P(i, k-i) /[k R(k)] .
$$

This will be zero for $x \leq x_{c}$, non-zero for $x>x_{c}$, where $x_{c}$ is the value of $x$ at which $P(i, k-i)$ first becomes two-peaked. Numerical studies up to $k=56$ are reported in Appendix D. They indicate that $x_{c} \simeq 9.5$, corresponding to $q_{c} \simeq 72$. Above this value there is a discontinuity in the first derivative of the limiting value $\mathcal{K}$ of $R(k)^{1 / k}$ across the line $x=y$ in the $(x, y)$ plane. This is a first-order transition.

For the regular lattices, the singularity in the free energy of the selfdual Potts model at $q=q_{c}=4$ is essential (going like the exponential of $\left.-1 / \sqrt{ }\left|q-q_{c}\right|\right)$ : all derivatives exist and are continuous, but the function is non-analytic [4, 6]. It would be interesting to determine if the same is true for this random model.

\section{Acknowledgement}

The author belatedly thanks Professor W.T. Tutte for introducing him to this problem, and for showing how the result (1) can be proved recursively. He also thanks V.V. Bazhanov for showing that the the formula for $p$ as a function of $v$ can be obtained from [3], and A.J. Guttmann for observations on what constitutes a solution in statistical mechanical problems.

\section{Appendix A}

Here we give the derivation of Liu's functional relation (20). If $A$ is any edge of a graph $\mathcal{G}$ that is not a loop or an isthmus, let $\mathcal{G}_{A}^{\prime}$ be the graph obtained from $\mathcal{G}$ by deleting the edge $A$, and $\mathcal{G}_{A}^{\prime \prime}$ the graph

obtained by identifying the two ends to form a single vertex. Then Tutte [30] gives the following theorem:

THEOREM 1.

$$
\chi(\mathcal{G} ; x, y)=\chi\left(\mathcal{G}_{A}^{\prime} ; x, y\right)+\chi\left(\mathcal{G}_{A}^{\prime \prime} ; x, y\right)
$$


Also, if $\mathcal{G}$ is the union of two subgraphs $\mathcal{H}_{1}$ and $\mathcal{H}_{2}$ having just one vertex in common (so $\mathcal{G}$ is separable), then Tutte also gives

\section{THEOREM 2.}

$$
\chi(\mathcal{G} ; x, y)=\chi\left(\mathcal{H}_{1} ; x, y\right) \chi\left(\mathcal{H}_{2} ; x, y\right)
$$

Tutte used these theorems to obtain a functional relation for the generating function of the sum of dichromatic polynomials over all rooted maps. Here we shall adapt his method to the sum over all non-separable rooted maps.

The only non-separable maps with a loop or an isthmus are the first two graphs in Figure 2. Applying Theorem 1 to all other maps, we obtain

$$
\Phi=x u_{1}^{2} u_{2} v_{2}+y u_{1} u_{2}^{2} v_{1}+Z_{1}+Z_{2}
$$

where

$$
\begin{aligned}
& Z_{1}=\sum \chi\left(M_{A}^{\prime} ; x, y\right) u_{1}^{m} u_{2}^{n} v_{1}^{i} v_{2}^{j}, \\
& Z_{2}=\sum \chi\left(M_{A}^{\prime \prime} ; x, y\right) u_{1}^{m} u_{2}^{n} v_{1}^{i} v_{2}^{j},
\end{aligned}
$$

the sums being over distinct non-separable rooted maps $M$ with at least two edges.

The map $M_{A}^{\prime}$ may be separable, but only in a very specific way: if $s$ is the root vertex of $M$ and $t$ the other end of the edge $A$, there may be a sequence of nodes through which any route (after deletion of the edge $A$ ) from $s$ to $t$ must pass. These, and only these, are vertices at which $M_{A}^{\prime}$ can be separated. For instance, in Figure 3 all routes from $s$ to $t$ (not along $A$ ) must pass sequentially through the two nodes $p$ and $q$. If $f$ is the root face of $M$, and $g$ the other face adjacent to $A$, then $p$ and $q$ are points where the faces $f$ and $g$ touch.

Consider the situation shown in Figure 3, where $M_{A}^{\prime}$ consist of three non-separable maps $M_{1}, M_{2}, M_{3}$ linked at $p$ and $q$. We ask how many ways three such given rooted maps can be combined as in the Figure. For $M_{1}$ we can take the root-vertex to be $s$, the root-face to be $g$, and the root-edge to be the first edge from $s$ alongside $g$. Similarly, $M_{2}$ has root-vertex $p$ and root-face $g ; M_{3}$ has $q$ and $g$. Let $m_{\alpha}, n_{\alpha}, i_{\alpha}, j_{\alpha}$ be the values of $m, n, i, j$ for $M_{\alpha}$. Also, let the number of edges of $M_{\alpha}$ adjacent to the face $g$ be $r_{\alpha}$. Then $m_{\alpha}>r_{\alpha} \geq 1$, 


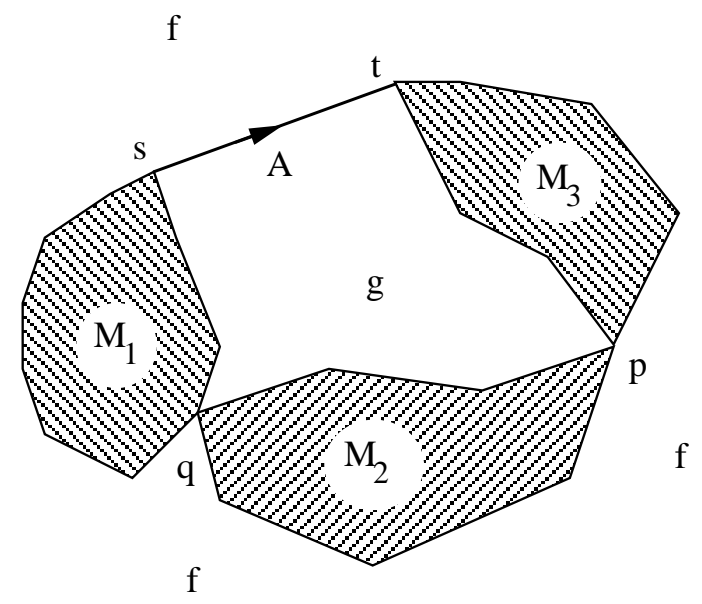

Figure 3: A map $M$ that separates after removal of the root edge $A$. Each shaded region represents a non-separable map with an outer face of at least two edges.

$n_{\alpha}>1$. Also, $m=m_{1}+m_{2}+m_{3}-r_{1}-r_{2}-r_{3}+1, n=n_{1}+1$, $i=i_{1}+i_{2}+i_{3}+1, j=j_{1}+j_{2}+j_{3}$. Using Theorem 2, the total contribution to $Z_{1}$ from the terms indicated by Figure 3 is therefore

$$
\begin{gathered}
u_{1} u_{2} v_{1} \sum_{M_{1}} \sum_{r_{1}=1}^{m_{1}-1} u_{1}^{m_{1}-r_{1}} u_{2}^{n_{1}} v_{1}^{i_{1}} v_{2}^{j_{1}} \chi\left(M_{1} ; x, y\right) \\
\sum_{M_{2}} \sum_{r_{2}=1}^{m_{2}-1} u_{1}^{m_{2}-r_{2}} v_{1}^{i_{2}} v_{2}^{j_{2}} \chi\left(M_{2} ; x, y\right) \sum_{M_{3}} \sum_{r_{3}=1}^{m_{3}-1} u_{1}^{m_{3}-r_{3}} v_{1}^{i_{3}} v_{2}^{j_{3}} \chi\left(M_{3} ; x, y\right)
\end{gathered}
$$

Note that $u_{2}$ occurs only in the outer factor and the first sum.

Strictly, the $M_{\alpha}$-sums should be restricted to non-separable maps $M_{\alpha}$ with $m_{\alpha} \geq 2$. However, the only non-separable map with $m<2$ is the second map (the loop-map) in Figure 2, with $m=1$. If $m_{\alpha}=1$, there are then no terms in the $r_{\alpha}$ summation, so the loop-map does not contribute and we can extend the summations to all non-separable maps.

Each $r_{\alpha}$ summation over $u_{1}^{m_{\alpha}-r_{\alpha}}$ gives a factor

$$
\frac{u_{1}-u_{1}^{m_{\alpha}}}{1-u_{1}}
$$

(Note that this vanishes for $m_{\alpha}=1$.) Inserting these, using (10) and 
writing $\Phi\left(u_{1}, u_{2}, v_{1}, v_{2}, x, y\right)$ simply as $\Phi\left(u_{1}, u_{2}\right)$, (A5) reduces to

$$
u_{1} u_{2} v_{1} C\left(u_{1}, u_{2}\right) C\left(u_{1}, 1\right)^{2},
$$

where $C\left(u_{1}, u_{2}\right)$ is defined by (19).

Figures like Figure 3, but with $k+1$ separable pieces instead of three, give the same contribution ( $\mathrm{A6}$ ), but with $C\left(u_{1}, 1\right)^{2}$ replaced by $C\left(u_{1}, 1\right)^{k}$. Summing over $k=0,1,2, \ldots$, we obtain

$$
Z_{1}=u_{1} u_{2} v_{1} C\left(u_{1}, u_{2}\right) /\left[1-C\left(u_{1}, 1\right)\right] .
$$

To calculate $Z_{2}$ we note that $Z_{2}$ is the dual of $Z_{1}$ and obtain immediately

$$
Z_{2}=u_{1} u_{2} v_{2} D\left(u_{1}, u_{2}\right) /\left[1-D\left(1, u_{2}\right)\right],
$$

where $D\left(u_{1}, u_{2}\right)$ is also defined by (19).

From (A3), we therefore obtain the functional relation (20). This is equation (4.17) of [23], $\mu, \nu, x, y, z, t$ therein being our $x, y, v_{2}, v_{1}, u_{1}, u_{2}$.

\section{Appendix B}

Let $R(k)$ be the number of rooted non-separable maps with $k$ edges, and $U(k)$ the number of unrooted ones. Then $R(1), R(2), \ldots=2,1,2,6$, $22,91,408,1938,9614,49335, \ldots$ and $U(1), U(2), \ldots=2,1,2,3,6,16$, $42,151,596,2605, \ldots$

Also let $\phi(k)$ be the Euler totient function, i.e. the number of integers less than $k$ that are relatively prime to $k$ (including 1 ). Thus $\phi(1), \phi(2), \ldots=1,1,2,2,4,2, \ldots$

Then Liskovets and Walsh 21] show that

$$
4 k U(k)=2 R(k)+\sum_{j \mid k} \phi(k / j)\left(9 j^{2}-9 j+2\right) R(j)+k c_{k} R\left(k^{\prime}\right),
$$

where

$$
\begin{array}{cl}
c_{k}=(k+1), & k^{\prime}=(k+1) / 2 \text { if } k \text { is odd }, \\
c_{k}=(3 k-4) / 4, & k^{\prime}=k / 2 \text { if } k \text { is even , }
\end{array}
$$

the sum being over all positive integers $j$ that divide $k$ and are less than $k$. 
Since this $R(k)$ is known, being given by (43) with $x=1$, the relation (B1) defines $U(k)$. The number of ways of rooting a given map is at most twice the number of edges (two directions for each edge), so

$$
R(k) \leq 2 k U(k) \quad .
$$

Numerically we observe that $2 k U(k) / R(k)$ decreases to unity exponentially fast, suggesting that this upper bound on $R(k)$ is attained asymptotically.

\section{Appendix C}

To solve the equations (34), set $u_{2}=1$ in the equation not involving $\hat{D}$ and write $t\left(u_{1}\right)$ for $T\left(u_{1}, 1, v_{1}, v_{2}\right)$. We can eliminate the function $\hat{C}$. Writing $u_{1}$ simply as $u$, we obtain the functional relation

$$
t(u)=\left[u v_{1}+t(u)\right]\left[u v_{2}+\frac{u t(1)-t(u)}{1-u}\right] .
$$

Writing $t(1)$ as $t_{1}$, this can be written as

$$
\begin{gathered}
t(u)^{2}+\quad\left(1-u+u^{2} v_{2}-u v_{2}+u v_{1}-u t_{1}\right) t(u)+ \\
u^{3} v_{1} v_{2}-u^{2} v_{1} v_{2}-u^{2} v_{1} t_{1}=0
\end{gathered}
$$

Given $t_{1}$, this is a quadratic equation for $t(u)$. Its discriminant is a quartic in $u$. We consider the situation when $v_{1}$ and $v_{2}$ are both small and of order $v$. This quartic then has two zeros close to one, and two of order $1 / v$.

The solution $t(u)$ of the quadratic is certainly analytic for sufficiently small $u$, so has a convergent Taylor expansion. For a given map $M$ (other than the link map) of type $(m, n, i, j), i \geq 1$ and $j \geq m-1$, so $i+j \geq m$. The coefficient of $u^{m}$ in the expansion of $t(u)$ is therefore no bigger than order $v^{m}$, so the radius of convergence is of order $1 / v$. It follows that the two zeros of the discriminant close to one must coincide, otherwise $t(u)$ would have a square root singularity at them.

This condition gives a fifth-degree equation for $t_{1}$ in terms of $v_{1}$ and $v_{2}$. We do not write it down here as it is far better to proceed as follows. As a polynomial in $u$, the discriminant has the form $1+$ $\cdots+v_{2}^{2} u^{4}$. It must therefore be identical to the polynomial (1$e u)^{2}\left(1+2 f u+s^{2} u^{2}\right)$, where $e, f, s$ are parameters to be determined. Equating coefficients of $u, \ldots, u^{4}$, we obtain four relations between 
$v_{1}, v_{2}, t_{1}, e, f, s$, the last of which can be written as $v_{2}=e s$. If we then define $p, r$ so that $e=1-p, s / e=r$, we find $f=r(2 p r-p-1)$ and that $v_{1}, v_{2}, t_{1}=T(1,1)$ are given by (35) and (36).

We thus have a parametrisation in terms of the variables $p, r$. Given $v_{1}, v_{2}$, we can in principle solve for $p, r$ and then obtain $T(1,1)$. We should choose the solution where $p \simeq v_{1}, q \simeq v_{2}, T(1,1) \simeq v_{1} v_{2}$ when $v_{1}, v_{2}$ are both small. Note that $T(1,1)$ is plainly a symmetric function of $v_{1}, v_{2}$, in agreement with duality.

Writing $u$ again as $u_{1}$, we can go on to solve for $t\left(u_{1}\right)$ by replacing $u_{1}$ by $w_{1}$, where

$$
u_{1}=\frac{w_{1}\left(1-p r w_{1}\right)}{1-r+r(1-p) w_{1}}
$$

As $w_{1}$ increases from 0 to $1, u_{1}$ also increases from 0 to 1 .

The discriminant of the quadratic is now a perfect square and we find

$$
t\left(u_{1}\right)=T\left(u_{1}, 1\right)=\frac{p r w_{1}^{2}\left[1-p-r+p r^{2}-p r(1-p) w_{1}\right]}{1-r+r(1-p) w_{1}},
$$

choosing the solution which vanishes when $u_{1}$ and $w_{1}$ are zero.

If we also define $w_{2}$ in terms of $u_{2}$ by

$$
u_{2}=\frac{w_{2}\left(1-p r w_{2}\right)}{1-p+p(1-r) w_{2}}
$$

then from the duality symmetry

$$
T\left(1, u_{2}\right)=\frac{p r w_{2}^{2}\left[1-p-r+p^{2} r-p r(1-r) w_{2}\right]}{1-p+p(1-r) w_{2}} .
$$

It is now easy to solve (33) - (34) for the full function $T\left(u_{1}, u_{2}\right)$ (the equations are linear in this function), giving

$$
\begin{gathered}
T\left(u_{1}, u_{2}\right)=\operatorname{pr} w_{1}^{2} w_{2}^{2}\left(1-p r w_{1}\right)\left(1-p r w_{2}\right) \times \\
\frac{(1-p)(1-r)-p r(1-p) w_{1}-p r(1-r) w_{2}}{\left(1-p r w_{1} w_{2}\right)\left[1-r+r(1-p) w_{1}\right]\left[1-p+p(1-r) w_{2}\right]} .
\end{gathered}
$$

This completes the parametric solution of the functional relation for $T\left(u_{1}, u_{2}\right)$, but it is still far from obvious that the coefficients in the expansion in powers of $v_{1}, v_{2}$ will be anything straightforward. In fact they are (at least for $T(1,1)$ ), as one can observe empirically. The key to the proof is the identities (39), (40). 
Let us look at the second (simpler) identity (40) first. The rhs is certainly some function of $m$ : write it as $P(m)$. If (35) is true, then $p$ is related to $v_{1}=v_{2}=v$ by $v=p(1-p)^{2}$. Multiplying by $p^{m}$, this implies the identity

$$
P(m+3)-2 P(m+2)+P(m+1)-v P(m)=0 .
$$

This is easily verified from the series expansion (40) of $P(m)$.

But (C8) is a third-order difference equation. For integer $m$ it follows that

$$
P(m)=A_{1} p_{1}^{m}+A_{2} p_{2}^{m}+A_{3} p_{3}^{m},
$$

where $p_{1}, p_{2}, p_{3}$ are the three roots of $v=p(1-p)^{2}$ and $A_{1}, A_{2}, A_{3}$ are some coefficients, independent of $m$.

If we write (C9) down for three successive values of $m$, i.e. $m, m+$ $1, m+2$, we can solve the resulting three equations for $A_{1}, A_{2}, A_{3}$ (provided $p_{1}, p_{2}, p_{3}$ are distinct, as they are for $|v|<4 / 27$ ). The result has the form

$$
A_{j}=\left[c_{1 j} P(m)+c_{2 j} P(m+1)+c_{3 j} P(m+2)\right] / p_{j}^{m},
$$

where the coefficients $c_{1 j}, c_{2 j}, c_{3 j}$ are independent of $m$.

For $v$ sufficiently small, we can bound the coefficients in the expansion (40) and show that $P(m)$ is of order $v^{m}$. The three roots of the cubic are close to $v, 1,1$; we choose $p_{1}=p \simeq v, p_{2} \simeq p_{3} \simeq 1$. Now let $m \rightarrow \infty$ in (C10): the rhs vanishes exponentially for $j=2,3$, so $A_{2}=A_{3}=0$. Hence $P(m)$ is proportional to $p_{1}^{m}=p^{m}$. From (40), $P(0)=1$, so $P(m)=p^{m}$ and we have proved (40) for sufficientl small $v$. Both sides exist and are analytic for $|v|<4 / 27$, so it is true throughout this domain.

When $n=1$ the theorem implies that

$$
p=\frac{2}{3}\left\{1-F\left(\frac{1}{3},-\frac{1}{3} ; \frac{1}{2} ; \frac{27 v}{4}\right)\right\},
$$

a result that can be obtained from equations (2.8.11) of [3]. For all $n$,

$$
P(n)=v^{n}{ }_{3} F_{2}\left(\frac{2 n}{3}, \frac{2 n+1}{3}, \frac{2 n+2}{3} ; n+\frac{1}{2}, n+1 ; \frac{27 v}{4}\right),
$$

${ }_{3} F_{2}$ being the generalized hypergeometric function (section 9.14.1 of Ref. 15]).

The first identity (39) can be proved in the same manner. One first eliminates $r$ between the equation (35) to obtain $v_{1}(1-p)^{4}=$ 


\begin{tabular}{|rr|rc|rc|}
\hline$k$ & $x_{c}$ & $k$ & $x_{c}$ & $k$ & $x_{c}$ \\
\hline 4 & 11.52079729 & 22 & 9.13390695 & 40 & 9.48204802 \\
6 & 9.10192128 & 24 & 9.21188431 & 42 & 9.49075809 \\
8 & 8.57757720 & 26 & 9.27606631 & 44 & 9.49647477 \\
10 & 8.49125873 & 28 & 9.32844518 & 46 & 9.49963733 \\
12 & 8.55621629 & 30 & 9.37088248 & 48 & 9.50061434 \\
14 & 8.67512255 & 32 & 9.40499415 & 50 & 9.49971657 \\
16 & 8.80618487 & 34 & 9.43213746 & 52 & 9.49720720 \\
18 & 8.93047698 & 36 & 9.45343658 & 54 & 9.49331008 \\
20 & 9.04035623 & 38 & 9.46981849 & 56 & 9.48821637 \\
\hline
\end{tabular}

Table 2: The value of $x_{c}$ for $k=4,6, \ldots, 56$.

$p\left[(1-p)^{2}-v_{2}\right]^{2}$. This is fifth degree equation for $p$. When $v_{1}, v_{2}$ are small the roots are approximately $v_{1}, 1,1,1,1$. Multiplying the equation by $p^{m}$ gives a fifth-order linear difference equation for the rhs $P(m, n)$ of (39), as a function of $m$. We can verify that it is satisfied by the given series expansion. Proceeding as before, it follows that $P(m, n) / p^{m}$ is independent of $m$. By symmetry, $P(m, n) /\left(p^{m} r^{n}\right)$ is independent of both $m$ and $n$. We can verify that $P(0,0)=1$, so $P(m, n)=p^{m} r^{n}$ and we have proved (39) for sufficiently small $v_{1}, v_{2}$. Both sides are analytic within the radius of convergence of the double series, so the identity is true in that domain.

\section{Appendix D}

For $x=y$ and $k=2 h$ an even integer, let $\gamma(k)$ be the relative difference between the central value (with $i=j=h$ ) of $P(i, j)$ and the adjacent value (with $i=h-1$ ), so $\gamma(k)=1-P(h-1, h+1) / P(h, h)$. This is a measure of the curvature of the function $P(i, k-i)$ : positive for $x<x_{c}$, negative for $x>x_{c}$. For $k$ finite we can define $x_{c}$ to be the value of $x$ for which $\gamma(k)$ vanishes. As $k$ increases we expect it to tend to a limit, namely the bulk value of $x_{c}$ referred to above.

We have calculated $x_{c}$ for $k=6, \ldots, 56$ and give the results in Table 2. They do indeed appear to be converging to a limit rather less than 
9.5 , but it is hard to be more precise than this. One would expect such values to converge monotonically to a limit as an inverse non-integer power law in $k$, but these initially decrease, then increase to a maximum at $k=48$, and then start to decrease again. It looks as though even higher values of $k$ are needed to examine the convergence. As a check of our numerical accuracy, we have performed the calculations to both 16-digit and 19-digit precision in Fortran (real*8 and real*12): the results agree to better than the accuracy of Table 2 .

\section{References}

[1] D. Arques, Une relation fonctionnelle nouvelle sur les cartes planaires pointées, J. Comb. Theory B 39 (1985) 27 - 42

[2] D. Arques, Enumeration des cartes pointées sur une surface orientable de genre quelconque en fonction des nombres de sommets et de faces, J. Comb. Theory B 77 (1999) $1-24$

[3] H. Bateman and Erdelyi, Higher Transcendental Functions, McGraw-Hill, New York, 1953

[4] R.J. Baxter, Potts model at the critical temperature, J. Phys. C 6 (1973) L445 - L448

[5] R.J. Baxter, H.N.V. Temperley and S.E. Ashley, Triangular Potts model at its transition temperature, and related models, Proc. Roy. Soc. A358 (1978) 535 - 559

[6] R.J. Baxter, Exactly solved models in Statistical Mechanics, Academic, London, 1982

[7] E.A. Bender and N.C. Wormald, The number of loopless planar maps, Discr. Math. 54235 - 237 (1985)

[8] E.A. Bender and L.Bruce Richmond, A survey of the asymptotic behaviour of maps, J. Comb. Theory B 40297 - 329 (1986)

[9] E. Brezin, C. Itzykson, G. Parisi and J.B. Zuber, Planar diagrams, Commun. Math. Phys. 59 (1978) $35-51$

[10] W.G. Brown, Enumeration of non-separable planar maps, Can. J. Math. 15526 - 545 (1963)

[11] W.G. Brown and W.T. Tutte, On the enumeration of rooted nonseparable planar maps, Can. J. Math. 16572 - 577 (1964) 
[12] J-M. Daul, $Q$-state Potts model on a random planar lattice, hepth/9502014 (1995)

[13] P. Di Francesco, B. Eynard and E. Guitter, Coloring random triangulations, Nucl. Phys. B516 (1998) 543 - 587

[14] C.M. Fortuin and P.W. Kasteleyn, On the random-cluster model. I. Introduction and relation to other models, Physica 57 (1972) $536-564$

[15] I.S. Gradshteyn and I.M. Ryzhik, Tables of Integrals, Series and Products, Academic, New York and London, 1965

[16] G. 't Hooft, A planar diagram theory for strong interactions, Nucl. Phys. B72 (1974) $461-473$

[17] L.P. Kadanoff, Some critical properties of the eight-vertex model, Phys. Rev. B 4 (1971) 3989 - 3993

[18] P.W. Kasteleyn and C.M. Fortuin, Phase Transitions in Lattice Systems with Random Local Properties, J. Phys. Soc. Japan, Suppl. 26 (1969) 11 - 14

[19] I.K. Kostov, Solvable statistical models on a random lattice, Nucl. Phys. B, Proc. Suppl. (Netherlands) 45A (1996) 13 - 28, hepth/9509124

[20] I. K. Kostov, Exact solution of the six-vertex model on a random lattice, Nuclear Physics B 575 (2000) 513 - 534

[21] V.A. Liskovets and T.R.S. Walsh, The enumeration of nonisomorphic two-connected planar maps, Can. J. Math. 35 (1983) $417-435$

[22] Y. Liu, Enumeration of rooted vertex non-separable planar maps, Chinese Annals of Mathematics 9 (1988) 390 - 403

[23] Y. Liu, On chromatic and dichromatic sum equations, Discr. Math. 84 (1990) 169 - 179

[24] Y. Liu, On functional equations arising from map enumerations, Discr. Math. 123 (1993) 93 - 109

[25] G. 't Hooft, A planar diagram theory for strong interactions, Nucl. Phys. B72 (1974) $461-473$

[26] W.T. Tutte, A census of planar maps, Can. J. Math. 15 (1963) $249-271$

[27] W.T. Tutte, On dichromatic polynomials, J. Comb. Theory 2 (1967) $301-320$ 
[28] W.T. Tutte, On the enumeration of planar maps, Bulletin Am. Math. Soc 74 (1968) $64-74$

[29] W.T. Tutte, On Chromatic Polynomials and the Golden Ratio, J. Comb. Theory 9 (1970) $289-296$

[30] W.T. Tutte, Dichromatic sums for rooted planar maps, Proceedings of Symposium in Pure Maths, Am. Math. Soc, 29 (1971) $235-245$

[31] W.T. Tutte, Dichromatic sums revisited, J. Comb. Theory B 66 (1996) $161-167$

[32] T.R.S. Walsh, Counting rooted maps by genus. III:Non-separable maps, J.Comb.Theory B 18 (1975) 222 - 259

[33] H. Whitney, The Colorings of Graphs, Ann. Math. (N.Y.) 33 (1932) $688-718$

[34] N.C. Wormald, On the number of planar maps, Can. J. Math. 33 (1981) 1 - 11

[35] F.Y. Wu, The Potts Model, Rev. Mod. Phys. 54 (1982) 235 - 268 